\title{
EPR spectra of spin labels in lipid bilayers
}

Hansgeorg Schindler and Joachim Seelig

Citation: The Journal of Chemical Physics 59, 1841 (1973); doi: 10.1063/1.1680269

View online: http://dx.doi.org/10.1063/1.1680269

View Table of Contents: http://aip.scitation.org/toc/jcp/59/4

Published by the American Institute of Physics

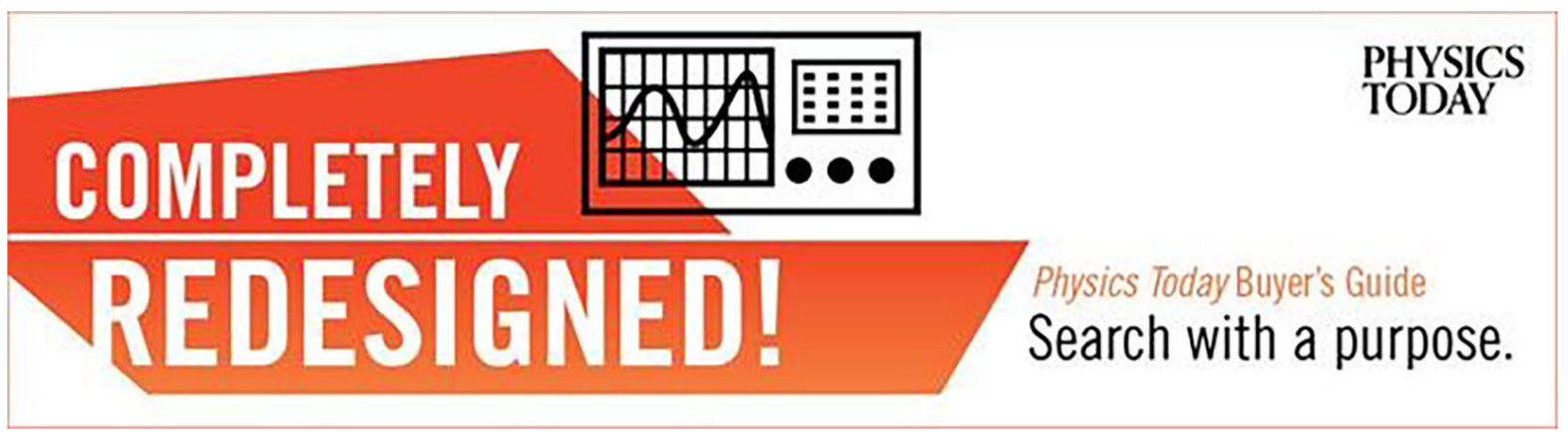




\title{
EPR spectra of spin labels in lipid bilayers
}

\author{
Hansgeorg Schindler and Joachim Seelig* \\ Biocenter of the University of Basel, Department of Biophysical Chemistry, Klingelbergstrasse 70, CH 4056 Basel, Switzerland
}

(Received 26 March 1973)

\begin{abstract}
Assuming a probability function $P(\theta) \alpha \exp \left\{(-q / R T) \cos ^{2} \Theta\right\}$ for the orientation of the chain segments in a lipid bilayer, a quantitative theory for the spin label spectra of such systems is developed. Only three parameters, namely two energy parameters $q_{3}$ and $q_{1}$ and the correlation time $\tau_{20}$ are required to yield perfect agreement between experimental and theoretical spectra for correlation times up to $3 \times 10^{-9} \mathrm{sec}$. In calculating the EPR spectra pseudosecular and nonsecular contributions to the linewidth are included, since especially the former exert a distinct influence on the spectra. Also discussed are the effects of tilt angle and spread angle. The theory is applied to the anisotropic motion of the fatty acid spin label $\mathrm{I}(13.2)$ in a decanol-sodium decanoate bilayer. Between 44 and $8{ }^{\circ} \mathrm{C}$ the correlation time is found to increase exponentially from $1.0 \times 10^{-10}$ to $4.0 \times 10^{-10} \mathrm{sec}$ with an activation energy of $6.84 \mathrm{kcal}^{\mathrm{mole}}{ }^{-1}$. The energy parameters $q_{3}$ and $q_{1}$, which determine the order parameters $S_{3}$ and $S_{1}$, remain constant in the same temperature range $\left(q_{3}=-2.9 \mathrm{kcal} \mathrm{mole}^{-1}\right.$; $q_{1}=1.7 \mathrm{kcal} \mathrm{mole}^{-1}$ ). The average orientation of the chain segment is perpendicular to the bilayer normal. Below $8^{\circ} \mathrm{C}$ a phase transition occurs and the chain segments become gradually tilted. At $-8^{\circ} \mathrm{C}$ the correlation time has increased to $2.8 \times 10^{-9} \mathrm{sec}$ and the chains are now tilted by approximately $18^{\circ}$ from the bilayer normal. From the correlation time the translational diffusion constant can also be deduced. At $21^{\circ} \mathrm{C}$ a value of $D_{\text {trans }}=1.5 \times 10^{-6} \mathrm{~cm}^{2} \mathrm{sec}^{-1}$ is obtained for the lipid molecules in the decanol-sodium decanoate bilayer. In vesicles of dimyristoyl-L- $\alpha$-lecithin at $21^{\circ} \mathrm{C}$ the analysis of the spectrum yields a correlation time of $2.5 \times 10^{-9} \mathrm{sec}$ and a diffusion constant of the lipid molecules of $D_{\text {trans }}=3.6 \times 10^{-8} \mathrm{~cm}^{2} \mathrm{sec}^{-1}$.
\end{abstract}

\section{INTRODUCTION}

There is now considerable evidence that a substantial portion of biological membranes contains a lipid bilayer. ${ }^{1}$ Magnetic resonance studies, in particular spin label investigations, have revealed that the membrane lipids are in a more or less "fluid" state. Probably the most important contributions to the membrane fluidity arise from fast rotations around the long molecular axes of the lipid molecules and from rotational isomerisations around carbon-carbon bonds. The latter process confers a distinct flexibility upon the fatty acid chains in a lipid bilayer as has been first demonstrated for very simple model systems. ${ }^{2}$ The alkyl chains were found to be well-ordered in the vicinity of the polar interface, but the motion became increasingly more random towards the central part of the bilayer. Such flexibility gradients have subsequently been observed in phospholipid bilayers ${ }^{3}$ and in biological membranes. ${ }^{4,5}$ They pose an interesting packing problem which has been overcome by the proposition of bent fatty acid chains in phospholipid bilayers. ${ }^{6,7}$ Nevertheless, the physical description of the chain conformation in lipid bilayers and related to this the question of interaction energies between adjacent alkyl chains are still in an early stage of discussion. ${ }^{8-11}$

Much could be gained in this situation if precise experimental parameters describing the behavior of the lipid molecules were available. This information can be obtained, in principle, from the
EPR signals of lipid spin labels, and the purpose of this report, therefore, is to reinvestigate the interpretation of such spectra. It will be shown in the following, that all molecular information is contained in three parameters, namely two energy parameters $q_{1}$ and $q_{3}$, which determine the average position of a chain segment, and a dynamic parameter, the correlation time $\tau_{20}$. These three parameters are sufficient to yield perfect agreement between experimental and simulated spectra for correlation times up to $3 \times 10^{-9} \mathrm{sec}$. The theory will be applied to various experimental situations: We shall discuss (1) the effect of various types of macroscopic alignments of the bilayer phases, (2) the influence of the order parameter, and (3) the temperature dependence of the spectra. The latter process will also yield the activation energy of the motion. This report is essentially concerned with the theory of the spin label spectra. The statistical-mechanical interpretation of the $q$ parameters together with results from other bilayer systems will be presented in a subsequent publication.

\section{THEORY OF THE SPIN LABEL SPECTRA}

The molecular motions in liquid crystals are highly anisotropic. The short-range order can be characterized by a director $z^{\prime}$ which defines the axis of rotational averaging. The average position of a molecule with respect to $z^{\prime}$ is then expressed in terms of order parameters $S_{i}{ }^{12}$ On a macroscopic scale, i. e., looking at a large sample, the $z^{\prime}$ axes of the microcrystalline regions are distrib- 
uted at random, but under certain experimental conditions a macroscopic alignment of the $z^{\prime}$ axes can be achieved. In the case of nematic phases this is possible by orienting the system in a strong magnetic field. For lamellar smectic phases the substance may be pressed between two closely spaced quartz plates, resulting in a homogeneous planar orientation. A cylindrical alignment of the bilayer phases can be obtained by orienting the system in glass capillaries. In an oriented planar sample the directions of the $z^{\prime}$ axes may be either identical with the axis of macroscopic orientation $N$ or they may be tilted by a certain angle. In simulating the spin label spectra one must also consider that the macroscopic ordering may not be perfect and that the $N$ axis of a planar sample may be varied arbitrarily with respect to the direction of the external magnetic field.

McFarland and McConnell presented a theory which takes into account most of the above mentioned phenomena. ${ }^{6}$ We have refined and extended their treatment by introducing essentially the following modifications. (1) We include nonsecular and pseudosecular terms in the linewidth calculation. These terms yield contributions for very short or very long correlation times up to $10^{-10}$ and $3 \times 10^{-9} \mathrm{sec}$, respectively. (2) The linewidth is expressed directly as a function of correlation times. (3) The order parameters are defined as functions of energy parameters. (4) The matrix of the order parameters is not necessarily assumed to be axial symmetric. A more detailed discussion of these and some other changes will be given in the following.

\section{A. Definition of Order Parameters}

A Cartesian coordinate system is ascribed to the NO radical such, that the $x$ axis is extended in the direction of the $\mathrm{N}-\mathrm{O}$ bond and the $z$ axis in the direction of the nitrogen $2 b \pi$ orbital. In the case of the fatty acid spin labels used in this work, the $z$ axis is practically identical with the direction of the extended hydrocarbon chain. The orientation of the NO group with respect to $z^{\prime}$ is then given by

$$
\begin{aligned}
& \mathbf{x} \cdot \mathbf{z}^{\prime}=\cos \theta_{1}(t)=\sin \beta(t) \cos \gamma(t), \\
& \mathbf{y} \cdot \mathbf{z}^{\prime}=\cos \theta_{2}(t)=\sin \beta(t) \sin \gamma(t), \\
& \mathbf{z} \cdot \mathbf{z}^{\prime}=\cos \theta_{3}(t)=\cos \beta(t) .
\end{aligned}
$$

$(\alpha, \beta$, and $\gamma$ are Eulerian angles, where $\alpha$ corresponds to a rotation around $z^{\prime}$. The use of Eulerian angles simplifies coordinate transformations.) The time averaged position of the NO group can then be expressed in terms of order parameters ${ }^{2} S_{i}$ :

$$
S_{i}=\frac{1}{2}\left(3\left\langle\cos ^{2} \theta_{i}\right\rangle-1\right), \quad i=1,2,3,
$$

with

$$
\sum_{i=1}^{3} S_{i}=0
$$

The calculation of the line shape parameters requires knowledge of averages $\left\langle\cos ^{4} \theta_{i}\right\rangle$, which are not accessible experimentally. Therefore, the following approximation will be made: From investigations of nematic liquid crystals it is known that the angular distribution of the molecules can be described by a probability function of the kind $^{13-16}$

$$
P(\theta) \propto \exp \left[-(q / R T) \cos ^{2} \theta\right],
$$

where $\theta$ is the angle between the long axis of the rodlike molecules and $z^{\prime}$. It is assumed that this probability function can also be applied to the orientation of hydrocarbon chains in lipid bilayers. Since the chains are flexible the energy parameters $q$ must vary as a function of the bond number $n$. It is further assumed that Eq. (4) not only describes the angular distribution of the long molecular axis but that similar relations hold for the remaining two axes. Combining Eqs. (2) and (4) we can thus write:

$S_{i}=-\frac{1}{2}+\frac{3}{2} \frac{\int_{0}^{\pi} \cos ^{2} \theta_{i} \exp \left[-\left(q_{i} / R T\right) \cos ^{2} \theta_{i}\right] \sin \theta_{i} d \theta_{i}}{\int_{0}^{\pi} \exp \left[-\left(q_{i} / R T\right) \cos ^{2} \theta_{i}\right] \sin \theta_{i} d \theta_{i}}$

As independent parameters we choose $q_{1}$ and $q_{3}$, which can be determined from $S_{1}$ and $S_{3}$. The third parameter is then fixed by Eq. (3). Knowing the $q_{i}$ parameters other angular averages are easily obtained.

\section{B. General Theory of the Spin Hamiltonian}

To proceed the total spin Hamiltonian $\hbar \mathcal{H C}(t)$ of the nitroxide radical is divided into a time-averaged part $\hbar \overline{\mathcal{K}}$ and a time-dependent part $\hbar \mathcal{H}_{1}(t)$ :

$$
\hbar \mathcal{K}(t)=\hbar \overline{\mathcal{K}}+\hbar \mathcal{K}{ }_{1}(t)=\hbar \overline{\mathfrak{K}}+\hbar\{\mathfrak{H C}(t)-\overline{\mathcal{K}}\} .
$$

$\hbar \overline{K C}$ determines the position of the EPR lines, while the perturbation $\hbar \mathcal{H}_{1}(t)$ causes relaxation and line broadening. This has been discussed extensively in various texts. $15,17-20$

$\hbar \mathcal{H C}(t)$ can be expressed as the sum of irreducible tensor operators $A_{\lambda}^{\prime \prime(l,-m)}$ :

$$
\hbar \mathcal{H C}(t)=\sum_{l, m, \lambda} F_{\lambda}^{(l,-m)} A_{\lambda}^{\prime \prime(l, m)}(t) .
$$

The double prime signifies that the $A_{\lambda}^{\prime \prime(l, m)}(t)$ are referred to the molecular axes of the NO radical. A listing of the tensor operators $A_{\lambda}^{(l, m)}$ and the spatial factors $F_{\lambda}^{(l,-m)}$ is given in Table $I$.

The transformation from the molecule fixed coordinates of the NO group to the space fixed coordinate system of the director $z^{\prime}$ is carried out 
with rotation matrices $D_{m^{2}, m}^{(t)}{ }^{21}$ :

$$
\hbar \mathcal{H C}(t)=\sum_{l, m, \lambda} F_{\lambda}^{(l,-m)} \sum_{p} D_{p, m}^{(l)}(\alpha \beta \gamma)(t) A_{\lambda}^{\prime(l, p)}
$$

The time dependence is now contained in the rotation matrices. A second transformation is necessary in order to rotate the coordinate system of the director $z^{\prime}$ into the laboratory system defined by the direction of the external magnetic field $H_{0}$ :

$$
\begin{aligned}
\hbar \mathcal{H}(l)=\sum_{l, m, \lambda} F_{\lambda}^{(l,-m)} \sum_{p} D_{p, m}^{(l)}(\alpha \beta \gamma)(t) \\
\quad \times \sum_{q} D_{q, p}^{(l)}\left(\alpha^{\prime} \beta^{\prime} \gamma^{\prime}\right) A_{\lambda}^{(l, q)} .
\end{aligned}
$$

$\beta^{\prime}$ denotes the angle between the magnetic field $H_{0}$ and the director axis $z^{\prime}$.

\section{Time Averaged Spin Hamiltonian}

The time averaged spin Hamiltonian must be invariant against rotations around the director axis $z^{\prime}$. Therefore

$\hbar \overline{\mathscr{K}}=\sum_{l, m, \lambda} F_{\lambda}^{(l,-m)} \overline{D_{0, m}^{(l)}(\beta \gamma)(t)} \sum_{q} D_{q, 0}^{(l)}\left(\alpha^{\prime} \beta^{\prime}\right) A_{\lambda}^{(l, q)}$.

For $z^{\prime} \| H_{0}\left(\beta^{\prime}=0^{9}\right) \mathrm{Eq}$. (10) reduces to

$$
\begin{aligned}
& \hbar \overline{\mathcal{K}}_{\|}=\sum_{l, m, \lambda} F_{\lambda}^{(l,-m)} D_{0, m}^{(l)}(\beta \gamma)(t) A_{\lambda}^{(l, 0)}, \\
& \hbar \overline{\mathcal{K}}_{\|}=\beta_{e} H_{0} g_{\|} S_{z}+\hbar I_{z} T_{\|} S_{z},
\end{aligned}
$$

with

$$
\begin{gathered}
g_{\|}=g+2 \Delta g S_{3}+\frac{2}{3} \delta g\left(S_{1}-S_{2}\right), \\
T_{\text {II }}=a+2 \Delta a S_{3}+\frac{2}{3} \delta a\left(S_{1}-S_{2}\right) .
\end{gathered}
$$

In deriving Eqs. (13) and (14) the terms $S^{ \pm} I^{\mp}$ have been neglected, since they lead only to small second-order effects.

\begin{tabular}{|c|c|}
\hline$A_{A}^{(1), n)}\left[S_{z} f_{z^{+}}+\frac{1}{2}\left(S^{+} \Gamma+S^{-} \tau^{+}\right)\right]$ & $F_{A}^{(n, 0)}=h a$ \\
\hline 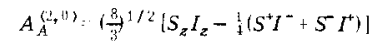 & $F_{A}^{(2,0)}=\left(\frac{3}{2}\right)^{1 / 2} h \Delta a$ \\
\hline$A_{A}^{(2, \pm 1)} \mp\left(S_{z} I^{ \pm}+S^{ \pm} I_{z}\right)$ & $F_{A}^{(2,+1):: 0}$ \\
\hline$A_{A}^{(2, \pm 2)} \quad S^{ \pm} I^{ \pm}$ & $F_{A}^{\left(2, x^{2}\right)}: \frac{1}{2} h \delta a$ \\
\hline$A_{G}^{(1), \cdots)} \cdot S_{z}$ & $F_{G}^{(1,0)}=h_{0} \beta_{e} g$ \\
\hline$A_{G}^{(1,1)}\left(\frac{3}{3}\right)^{1 / 2} S_{z}$ & $F_{G}^{(2,0)}\left(\frac{3}{2}\right)^{1 / 2} H_{0} \beta_{e} \Delta r$ \\
\hline$A_{G}^{(!, \pm 1)} \mp S^{ \pm}$ & $F_{G}^{(2, \pm 1)} \cdot 0$ \\
\hline$\left.A_{G}^{(1,}, \pm ?\right) 0$ & $F_{G}^{(2, \pm 2)} \cdot \frac{1}{2} H_{0} \beta_{e} \delta g r$ \\
\hline$a=\frac{1}{\mathfrak{j}}\left(T_{x x}+T_{y y}+T_{z z}\right)$ & $g: \frac{1}{3}\left(g_{x x}+g_{y y}+g_{z z}\right)$ \\
\hline$\Delta / y=\frac{1}{6}\left(2 T_{z z}-T_{x x}-T_{y y}\right)$ & $\Delta y=\frac{1}{6}\left(2 g_{z z}-g_{y y}-g_{x x}\right)$ \\
\hline$\delta a=\frac{1}{2}\left(T_{x x}-7_{y y}\right)$ & $\delta g=\frac{1}{2}\left(g_{x x}-g_{y y}\right)$ \\
\hline
\end{tabular}

For $z^{\prime} \perp H_{0}\left(\beta^{\prime}=90^{\circ}\right)$ the following result is obtained:

$$
\hbar \overline{\mathfrak{H}}_{\perp}=\sum_{l, m, \lambda} F_{\lambda}^{(l,-m)} \overline{D_{0, m}^{(l)}(\beta \gamma)(t)} D_{0,0}^{(l)}\left(\beta^{\prime}=90^{0}\right) A_{\lambda}^{(l, 0)} \text {, }
$$

TABLE I. Irreducible tensor operators $A_{\lambda}^{(t, m)}$ and spatial factors $F_{\lambda}^{(d,-m)}$.

$$
\begin{aligned}
& \hbar \overline{\mathcal{G}}_{1}=\beta_{e} H_{0} g_{\perp} S_{z}+\hbar I_{z} T_{\perp} S_{z}, \\
& g_{\perp}=g-\Delta g S_{3}-\frac{1}{3} \delta g\left(S_{1}-S_{2}\right), \\
& T_{\perp}=a-\Delta a S_{3}-\frac{1}{3} \delta a\left(S_{1}-S_{2}\right) .
\end{aligned}
$$

If $\beta^{\prime}$ lies between these two extremes, then

$$
\overline{\hbar \mathcal{H C}\left(\beta^{\prime}\right)}=\beta_{e} H_{0} g\left(\beta^{\prime}\right) S_{z}+\hbar I_{z} T\left(\beta^{\prime}\right) S_{z},
$$

with

$$
\begin{aligned}
& g\left(\beta^{\prime}\right)=\left[\cos ^{2} \beta^{\prime} g_{\|}^{2}+\sin ^{2} \beta^{\prime} g_{1}^{2}\right]^{1 / 2}, \\
& T\left(\beta^{\prime}\right)=\left[\cos ^{2} \beta^{\prime} T_{\|}^{2}+\sin ^{2} \beta^{\prime} T_{1}^{2}\right]^{1 / 2} .
\end{aligned}
$$

Compared to Eq. (10) the angle $\alpha^{\prime}$ is eliminated from Eq. (19). Insertion of Eqs. (13), (14), (17), (18) into (20) and (21) and in combination with (19) finally yields the effective spin Hamiltonian as a function of $S_{1}, S_{2}$, and $\beta^{\prime}$.

The last problem in the theory of the static Hamiltonian is to include the effect of tilted fatty acid chains. ${ }^{6,7}$ A tilt angle can be detected only in an oriented sample of bilayers (i.e., planar or cylindrical orientation) but is averaged out in a random distribution. We therefore confine our discussion to a planar oriented sample. The normal on the bilayer planes is denoted with $N$. If $\vartheta$ is the tilt angle between $N$ and the director axis $z^{\prime}$ and if $\Delta$ is the angle between $N$ and the magnetic field $H_{0}$, then $\beta^{\prime}$, the angle between $z^{\prime}$ and $H_{0}$, is found by the following relation :

$$
\cos \beta^{\prime}=\cos \vartheta \cos \Delta+\sin \vartheta \sin \Delta \cos \varphi .
$$

All director axes need not have exactly the same tilt angle, instead they may be distributed according to a certain probability function $p(\vartheta)$. In our calculations we have used a distribution function suggested by McFarland and McConnell, ${ }^{6}$ but other choices are equally possible:

$$
p(\vartheta) \sim \sin \vartheta \exp \left[-(\vartheta-\bar{\vartheta})^{2} / 2 \vartheta_{0}^{2}\right] .
$$

$\bar{\vartheta}$ is the average angle of tilt and $\vartheta_{0}$ is called the spread angle. In order to simulate the EPR spectra Eq. (22) is inserted into Eqs. (20) and (21). The angles $\vartheta$ and $\varphi$ are then varied between $0 \leq \vartheta$ $\leq \pi$ and $0 \leq \varphi \leq 2 \pi$ and each spectrum for a given $\vartheta$ is weighted by Eq. (23).

\section{Linewidth of the Spin Label Spectra}

The linewidth of an electron spin resonance signal depends on both the molecular tumbling rate and the orientation of the radical with respect to the external magnetic field. In the case of the nitroxide radical (electron spin $\frac{1}{2}$ ) the linewidth parameter $T_{2}$ can be calculated according to Eq. $(24)^{15}$ :

$T_{2}^{-1}$

$=\int_{0}^{t \gg \tau} c \overline{\left\langle M, \frac{1}{2}\left|\left[\mathcal{H C}_{1}^{*}(t),\left[\mathcal{K}_{1}^{*}(t-\tau), S^{+}\right]\right]\right|-\frac{1}{2}, M\right\rangle} d \tau$, 


$$
\mathfrak{H}_{1}^{*}(t)=\exp (-i \overline{\mathfrak{F}} t) \mathcal{H}_{1}(t) \exp (i \overline{\mathscr{H}} t) .
$$

If $\mathcal{H}_{1}(t)$ is expanded in irreducible tensor operators the following result is obtained after two coordinate transformations:

$$
\hbar \mathcal{F}_{1}(t)=\sum_{l, m, \lambda} F_{\lambda}^{(l,-m)} \sum_{p}\left\{D_{p, m}^{(l)}(\alpha \beta \gamma)(t)-\overline{D_{p, m}^{(l)}(\alpha \beta \gamma)}\right\} \sum_{q} D_{q, p}^{(l)}\left(\alpha^{\prime} \beta^{\prime} \gamma^{\prime}\right) A_{\lambda}^{(l, a)}
$$

Taking into account the rotational symmetry with respect to $z^{\prime}$ the commutator brackets in (24) can be simplified to give:

$$
\begin{aligned}
\overline{\left\langle M, \frac{1}{2}\left|\left[\mathcal{H}_{1}^{*}(t),\left[\mathcal{H}_{1}^{*}(t-\tau), S^{+}\right]\right]\right|-\frac{1}{2}, M\right\rangle}= & \sum_{\substack{m, \lambda \\
m^{0}, \lambda^{\prime}}} F_{\lambda}^{(2,-m)} F_{\lambda^{\prime}}^{\left(2,-m^{\prime}\right)} \\
& \times \sum_{p}\left\{\overline{D_{p, m}^{(2) *}(\beta \gamma)(t) D_{p,-m^{\prime}}^{(2)}(\beta \gamma)(t-\tau)}-\overline{D_{p, m}^{(2) *}(\beta \gamma)} \overline{\left.D_{p,-m^{\prime}}^{(2)}(\beta \gamma)\right\}}\right. \\
& \times \sum_{a}\left|D_{q, p}^{(2)}\left(\beta^{\prime}\right)\right|^{2}\left|\left\langle M, \frac{1}{2}\left|\left[A_{\lambda}^{*(2, \alpha)}(t),\left[A_{\lambda^{\prime}}^{*(2,-q)}(t-\tau), S^{+}\right]\right]\right|-\frac{1}{2}, M\right\rangle\right| .
\end{aligned}
$$

The second transformation is determined by the absolute value of the rotation matrices $D_{q, p}^{(2)}$ and thus only depends on $\beta^{\prime}$, the angle between $H_{0}$ and $z^{\prime}$. Eq. (27) differs from previous results ${ }^{6,15,20}$ in that no assumptions have been made about special symmetries of the molecule fixed tensors. The linewidth is therefore a function of two rotation angles $(\beta, \gamma)$, i.e., two order parameters $\left(S_{3}, S_{1}\right)$. The correlation between the Wigner rotation matrices can be approximated by an anisotropic Brownian diffusion model, ${ }^{18}$

$$
\begin{aligned}
& \overline{D_{p, m}^{(2) *}(\beta \gamma)(t) D_{p,-m^{\prime}}^{(2)}(\beta \gamma)(t-\tau)} \\
& =\overline{D_{p, m}^{(2)} *} \overline{(\beta \gamma) D_{p,-m^{0}}^{(2)}(\beta \gamma)} \exp \left(-|\tau| / \tau_{2 m}\right) .
\end{aligned}
$$

After some algebra this leads to the final linewidth formula

$$
\begin{aligned}
T_{2}^{-1}= & \hbar^{2} \sum_{\substack{m, \lambda \\
m^{\prime}, \lambda^{\prime}}} F_{\lambda}^{(2,-m)} F_{\lambda^{\prime}}^{\left(2,-m^{\prime}\right)} \sum_{p} \overline{\left(D_{p, m}^{(2) *}(\beta \gamma) D_{p,-m^{\prime}}^{(2)}(\beta \gamma)\right.} \\
& \left.-\overline{D_{p, m}^{(2) *}(\beta \gamma)} \overline{D_{p,-m^{\prime}}^{(2)}(\beta \gamma)}\right) \\
& \quad \times \sum_{q}\left|D_{q, p}^{(2)}\left(\beta^{\prime}\right)\right|^{2}\left|j\left(\lambda q, \lambda^{\prime}-q\right)\right| \cdot \quad \text { (29) }
\end{aligned}
$$

The spectral densities are listed in Table II. (In evaluating the $j\left(\lambda q, \lambda^{\prime}-q\right)$ nonsecular contributions to $\overline{\mathfrak{H}}$ have been ignored. ${ }^{15}$ ) Eq. (29) is a general expression for the linewidth of the nitroxide radi$\mathrm{cal}$, in which pseudosecular and nonsecular terms are included. Eq. (29) also allows for the possibility of different correlation times for the three different axes of molecular rotation (anisotropic diffusion tensor). Since the nuclear spin is not quantized exactly along $H_{0}$, the following correction factor $^{6}$ can be used for the $A_{\lambda}^{(2, \alpha)}$ :

$$
\cos \beta^{\prime \prime}=\frac{T_{\|} \cos ^{2} \beta^{\prime}+T_{1} \sin ^{2} \beta^{\prime}}{\left[T_{11}^{2} \cos \beta^{\prime}+T_{1}^{2} \sin \beta^{\prime}\right]^{1 / 2}}
$$

\section{COMPARISON BETWEEN EXPERIMENTAL AND SIMULATED SPIN LABEL SPECTRA}

\section{A. Experimental}

The applicability of the preceding theory will now be demonstrated for the case of label $\mathrm{I}(13,2)$ :

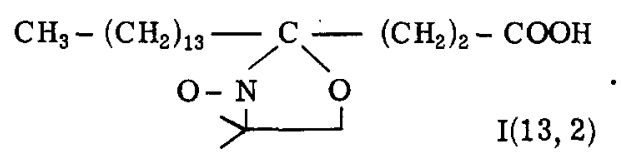

This label has been chosen, since its motion in a bilayer is highly anisotropic and the simulation of such spectra is the most critical test of our theory. $I(13,2)$ was incorporated into a liquid crystalline

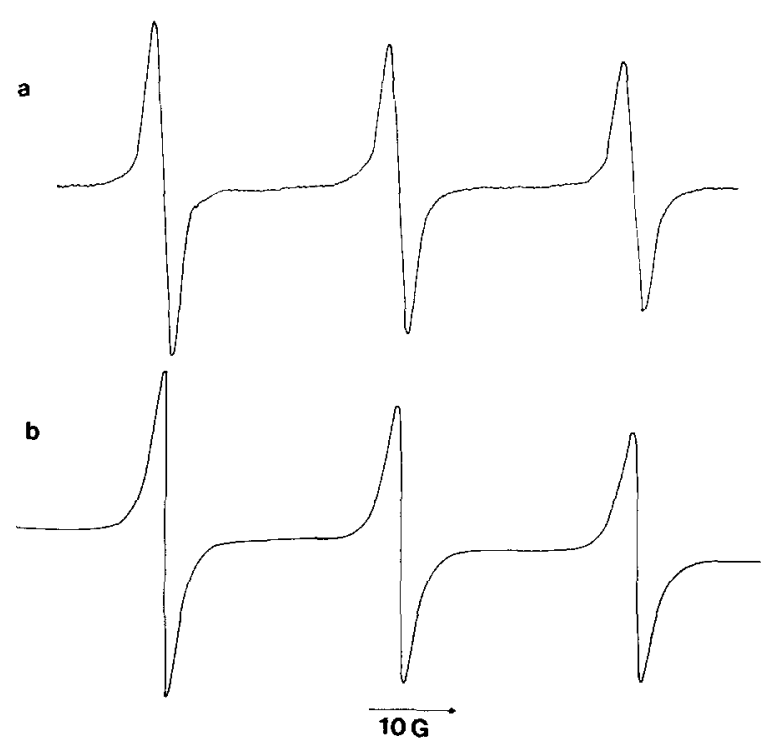

FIG. 1. Spin label I(13.2) in a planar oriented sample of bilayers at $21^{\circ} \mathrm{C}$. Magnetic field $H_{0}$ parallel to bilayer normal $N$. (a) experimental spectrum, (b) calculated spectrum with $q_{3}=-2.895 \mathrm{kcal} / \mathrm{mole}, q_{1}=1.684$ $\mathrm{kcal} / \mathrm{mole}, \tau_{20}=2.3 \times 10^{-10} \mathrm{sec}, \vec{\vartheta}=0^{\circ}, \vartheta_{0}=3.4^{\circ}$ Residual linewidth: $b=0.65 \mathrm{G}, c=0.55 \mathrm{G}$. 
TABLE II. Spectral densities.

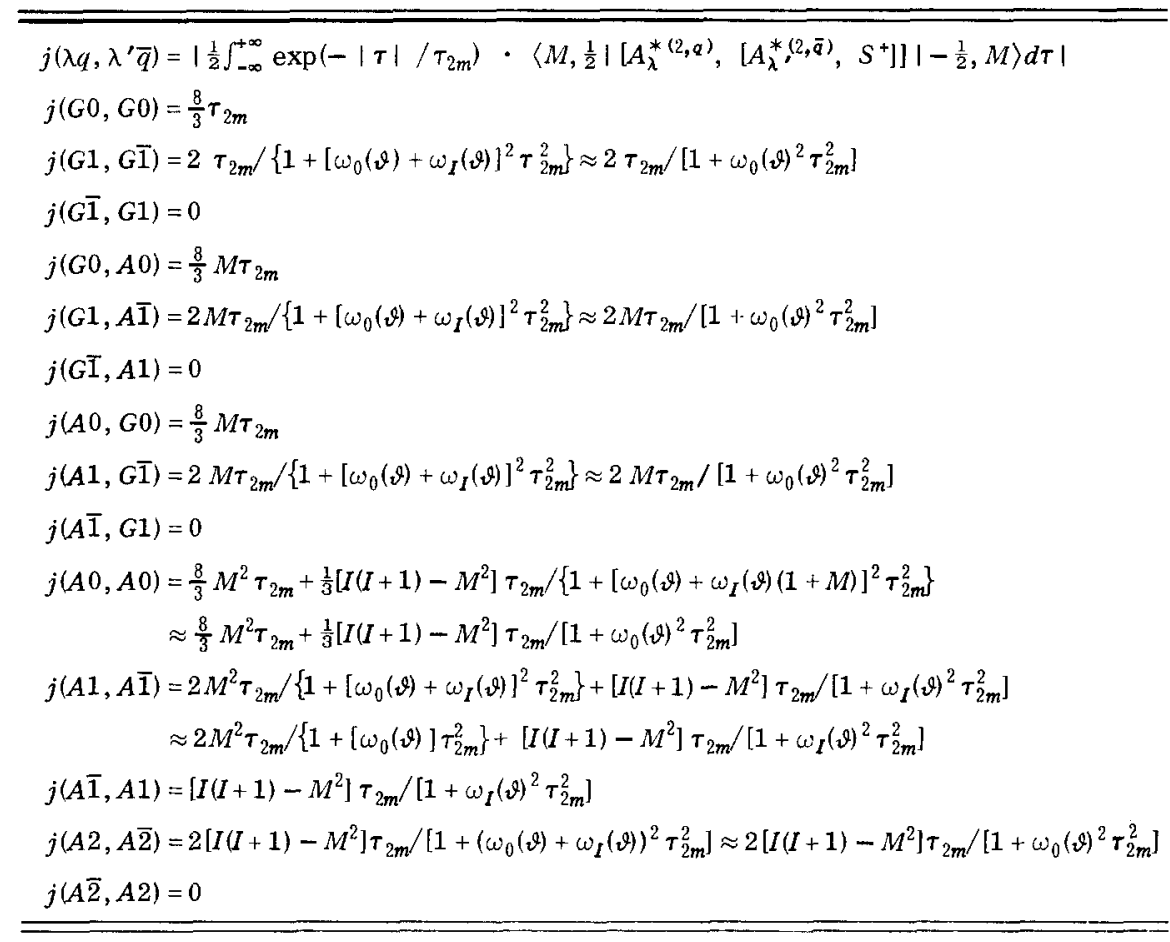

bilayer with the following chemical composition: Decanol (42 wt\%), sodium decanoate (28wt\%), and water $(30 \mathrm{wt} \%)$. The synthesis of the spin label and its motion in this bilayer phase have been described elsewhere. ${ }^{2,8,9}$ The concentration of spin label was approximately $0.1 \%$ by weight.

The theoretical discussion has made it clear that for constant order parameters $S_{i}$ and constant correlation times $\tau_{2 m}$ the spectrum should still be influenced profoundly by the macroscopic ordering of the bilayers. We have therefore investigated three different types of bilayer orientations: (1) a planar arrangement, induced by introducing the sample between two optically flat quartz plates (spacing $0.01 \mathrm{~mm}$ ). The magnetic field was then applied parallel and perpendicular to the normal of the bilayer surfaces. The corresponding spectra are shown in Fig. 1(a) and Fig. 2(a), respectively. (2) A cylindrical ordering, obtained by sucking the liquid crystalline phase into thin capillaries (with a diameter of approximately $0.04 \mathrm{~mm}$ ). This situation bears some resemblance to the arrangement of the lipid bilayers in the myelin sheath of a nerve fiber. In order to increase the signal intensity, approximately 15 capillaries of $2 \mathrm{~cm}$ length were bundled together. This package of parallel glass fibers was mounted on a teflon rod and inserted into the resonance cavity so that the magnetic field was perpendicular to the cylinder axes. Figure 3(a) shows the experimental spectrum.

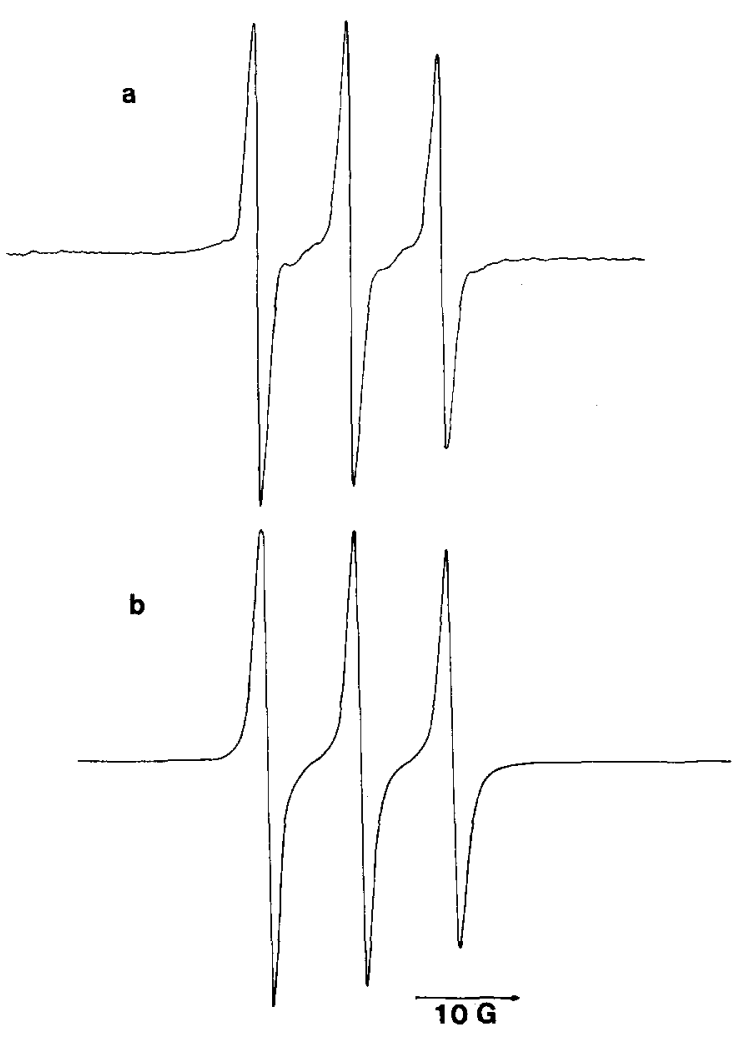

FIG. 2. Spin label I(13.2) in a planar oriented sample of bilayers at $21^{\circ} \mathrm{C}$. Magnetic field $H_{0}$ perpendicular to bilayer normal $N$. (a) experimental; (b) calculated spectrum. Same parameters as in Fig. 1. 


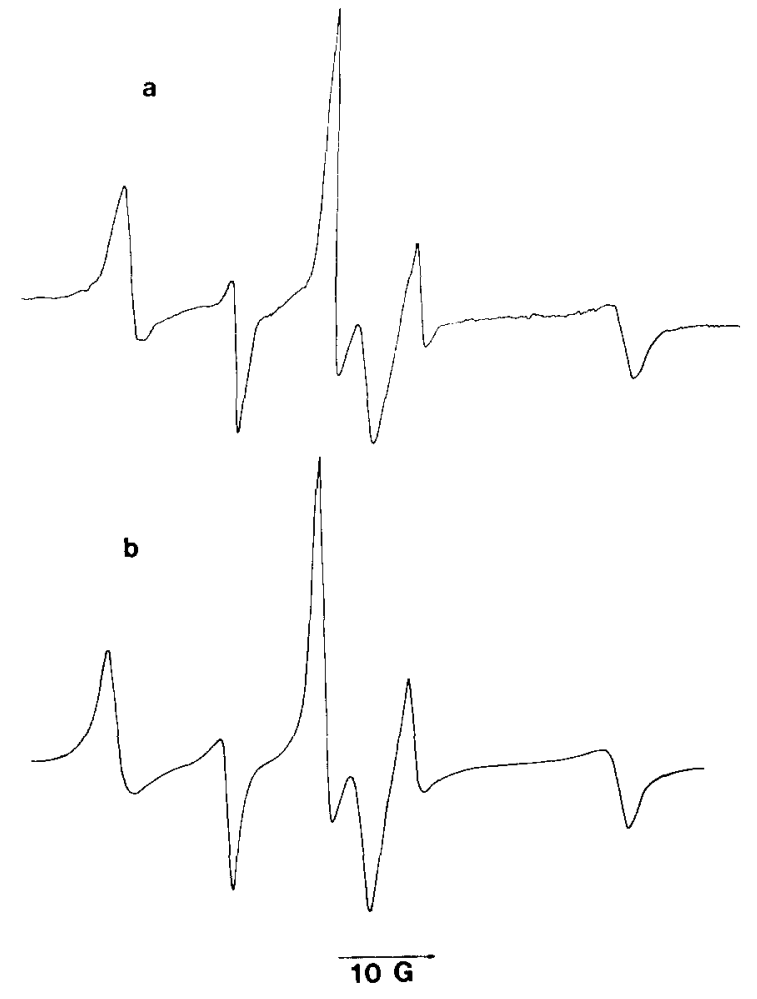

FIG. 3. Spin label I(13.2) in a cylindrical arrangement of bilayers at $21{ }^{\circ} \mathrm{C}$. (a) experimental; (b) calculated spectrum. Same parameters as in Fig. 1.

(3) A random distribution of microcrystalline bilayer regions, obtained by filling the material into a sample tube of rather large diameter $(d \sim 4 \mathrm{~mm})$. In this case small contributions from cylindrical orientation on the glass surfaces can be neglected compared to the bulk material. The spectrum is given in Fig. 4(a).

The EPR measurements were performed with a Varian E-9 spectrometer $(\sim 9.35 \mathrm{GHz}$ resonance frequency; $100 \mathrm{KHz}$ modulation frequency) equipped with a variable temperature unit. The temperature was measured by means of a thermistor inserted into the center of the cavity. The absolute temperatures are accurate to $\pm 1^{\circ} \mathrm{C}$.

\section{B. Simulated EPR Spectra}

The simulation of the spin label spectra was based on Lorentzian line shapes. We have also tried Gaussian lines, but these yielded unsatisfying results.

The residual linewidth $X$ was approximated by the following expression ${ }^{22}$

$$
X=b+c \cos ^{2} \beta^{\prime} .
$$

The influence of the parameters $b$ and $c$ on the spectrum was distinctly different from that of the order parameters or correlation times. There- fore $b$ and $c$ could be fitted after the other parameters had been optimized.

No a priori predictions can be made about the ratio of the correlation times $\tau_{2 m}$. In the case of label $\mathrm{I}(13,2)$ the best fit of the experimental data was obtained by assigning $\tau_{22} / \tau_{20}=1$.

The theoretical spectra depend strongly on the correct choice of the molecule fixed components of the $g$ and $T$ tensors. Since these are determined by the particular environment of the spin label, single crystal data must be corrected for the change of polarity in the bilayer membrane. In our simulation we have used the following parameters ${ }^{3,22-24}$ :

$$
\begin{array}{cl}
g_{x x}=2.00872, & g_{y y}=2.00616, \\
T_{x x}=6.95 \mathrm{G}, & T_{y y}=5.35 \mathrm{G}, \quad T_{z z}=33.00270,
\end{array}
$$

These numbers are within the limits of error of single crystal measurements. It should be pointed out here, that the spectra are especially sensitive to changes in $\delta a=\frac{1}{2}\left(T_{x x}-T_{y y}\right)$.

Spectra calculated on the basis of the foregoing assumptions are shown in Figs. 1(b)-4(b). All spectra were computed with the parameters $q_{i}$ and $\tau_{20}$ given in Fig. 1. In the cases of the random distribution and the planar oriented samples (both orientations) we have also measured the temperature dependence of the bilayer phase in the range of

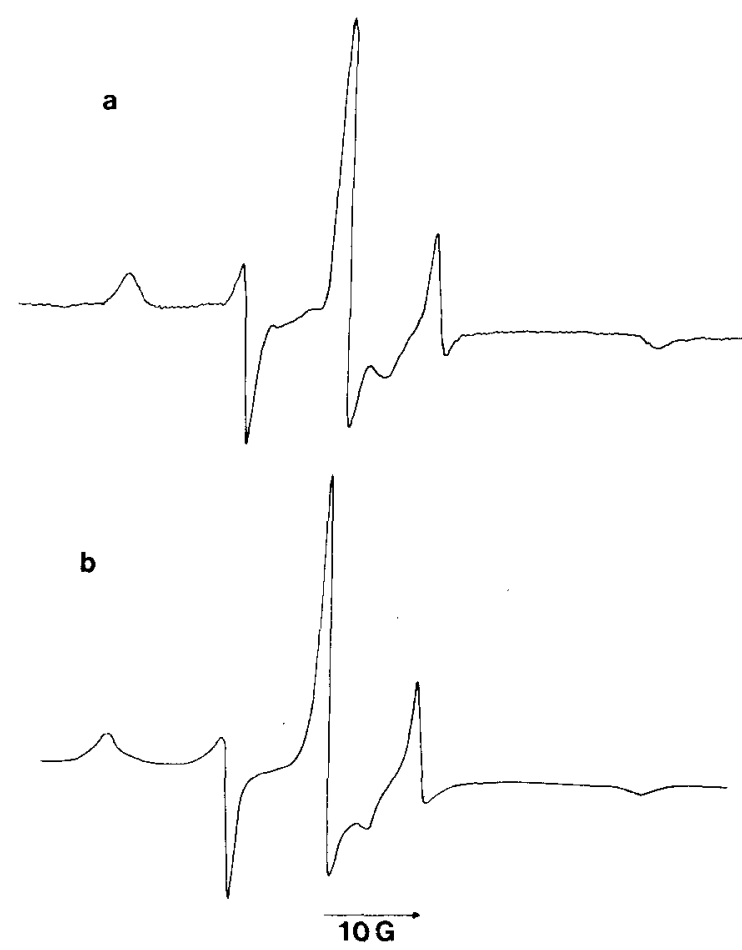

FIG. 4. Spin label I(13.2) in random distribution of bilayers at $21^{\circ} \mathrm{C}$. (a) experimental, (b) calculated spectrum. Same parameters as in Fig. 1. 
TABLE III. Molecular parameters for the motion of spin label I(13.2).

\begin{tabular}{|c|c|c|c|c|}
\hline & \multicolumn{4}{|c|}{ Temperiture $\left({ }^{\circ} \mathrm{C}\right)$} \\
\hline & 44.2 & 21.5 & 8.4 & -8.4 \\
\hline $\begin{array}{l}\text { Correlation time } \\
\tau_{20} \cdot 10^{10}(\mathrm{sec})\end{array}$ & $1.0+0.2$ & $2.3 \pm 0.2$ & $4.0 \pm 0.2$ & $28 \pm 1$ \\
\hline $\begin{array}{l}\text { Energy parameters } \\
-q_{3}(\mathrm{cal} / \mathrm{mole}) \\
+q_{1}(\mathrm{cal} / \mathrm{mole})\end{array}$ & $\begin{array}{l}2880 \\
1669\end{array}$ & $\begin{array}{l}2895 \\
1684\end{array}$ & $\begin{array}{l}2901 \\
1709\end{array}$ & $\begin{array}{l}3016 \\
1390\end{array}$ \\
\hline $\begin{array}{l}\text { Order parameters } \\
S_{3} \\
S_{1} \\
S_{2}\end{array}$ & $\begin{array}{r}0.61 \\
-0.25 \\
-0.36\end{array}$ & $\begin{array}{r}0.64 \\
-0.27 \\
-0.37\end{array}$ & $\begin{array}{r}0.66 \\
-0.28 \\
-0.38\end{array}$ & $\begin{array}{r}0.70 \\
-0.25 \\
-0.45\end{array}$ \\
\hline Tilt angle & $0^{\circ}$ & $0^{\circ}$ & $0^{\circ}$ & $18^{\circ}$ \\
\hline Spread angle & $7.0^{\circ}$ & $3.4^{\circ}$ & $5.0^{\circ}$ & $6.0^{\circ}$ \\
\hline $\begin{array}{l}\text { Isotropic hyperfine } \\
\text { splitting constant } \\
a(G)\end{array}$ & 15.0 & 15.0 & 15.0 & 15.24 \\
\hline
\end{tabular}

-8 to $+44^{\circ} \mathrm{C}$. The agreement between the computer fitted and the experimental spectra was practically perfect over this temperature interval. The results of our theoretical analysis for four different temperatures are summarized in Table III.

\section{DISCUSSION}

Inspection of Figs. 1-4 shows excellent agreement of the simulated spin label spectra with the experimental spectra. It should be emphasized, however, that these results can only be obtained if the time-averaged and the dynamic parts of the spin Hamiltonian are evaluated in their most general form. Since a concise discussion of the influence of the various parameters on the appearance of the EPR spectrum is rather difficult, only a representative example shall be given here. In Fig. 5 the spectra of label I(13.2) have been calculated with and without the inclusion of pseudosecular terms. It is obvious that pseudosecular terms contribute essentially to the over-all linewidth. In the case of Fig. 5(b) they account for approximately $30 \%$ of the intensity of the low and high field lines. Other significant terms which cannot be neglected are the $\delta a$ parameter or the order parameter $S_{1}$.

Let us now consider the structural and dynamic information contained in the line shape parameters. The correlation times $\tau_{20}\left(=\tau_{22}\right)$ are given in the first row of Table III. To the best of our knowledge this is the first accurate measurement of these correlation times in a lipid bilayer. In the range of 44 to $8{ }^{\circ} \mathrm{C}, \tau_{20}$ shows an exactly exponential temperature dependence with an activation energy of $E_{a}=6.84 \mathrm{kcal} \mathrm{mole}^{-1}$. The activation energies for the rotation of small molecules in the liquid state lie in the range of 2 to $4 \mathrm{kcal} \mathrm{mole}{ }^{-1}$, but for $n$-decane and tridecane, which have some structural relationship to the lipids of this bilayer, activation energies of 4.9 and $5.5 \mathrm{kcal} \mathrm{mole}^{-1}$, respectively, have been found close to the melting points of these paraffins. ${ }^{25}$ Since the flexibility gradient in lipid bilayers is caused by rotational isomerizations of carbon-carbon bonds, it may be speculated that the observed activation energy and also the equality $\tau_{20}=\tau_{22}$ are due to the same mechanism.

The viscosity in the bilayer as sensed by the spin label can be estimated according to

$$
\eta=3 k T \tau_{20} / 4 \pi r^{3}
$$

At the position of the NO group the radius of the spin labeled hydrocarbon chain is approximately $3.6 \AA$. With $\tau_{20}=2.3 \times 10^{-10} \mathrm{sec}$ we obtain a viscosity of $\eta=4.8 \times 10^{-2} \mathrm{P}$ at $21^{\circ} \mathrm{C}$. The diffusion constant for translational diffusion can also be calculated. For a decanol molecule of roughly $10 \AA$ length and $2.5 \AA$ radius this calculation yields $D_{\text {trans }}=1.5 \times 10^{-6}$ $\mathrm{cm}^{2} \mathrm{sec}^{-1}$.

We also incorporated label I (13.2) into vesicles of dimyristoyl- $\mathrm{L}-\alpha$-lecithin. At $21.5^{\circ} \mathrm{C}$ (this is $2{ }^{\circ} \mathrm{C}$ below the transition temperature of the gel $\rightarrow$ liquid crystal phase transition) we found a correlation time $\tau_{20}=2.5 \times 10^{-9} \mathrm{sec}$, which yields a viscosity of $\eta=0.5 \mathrm{P}$ and a translational diffusion constant for the lipid molecules of $D_{\operatorname{trans}}=3.6 \times 10^{-8}$
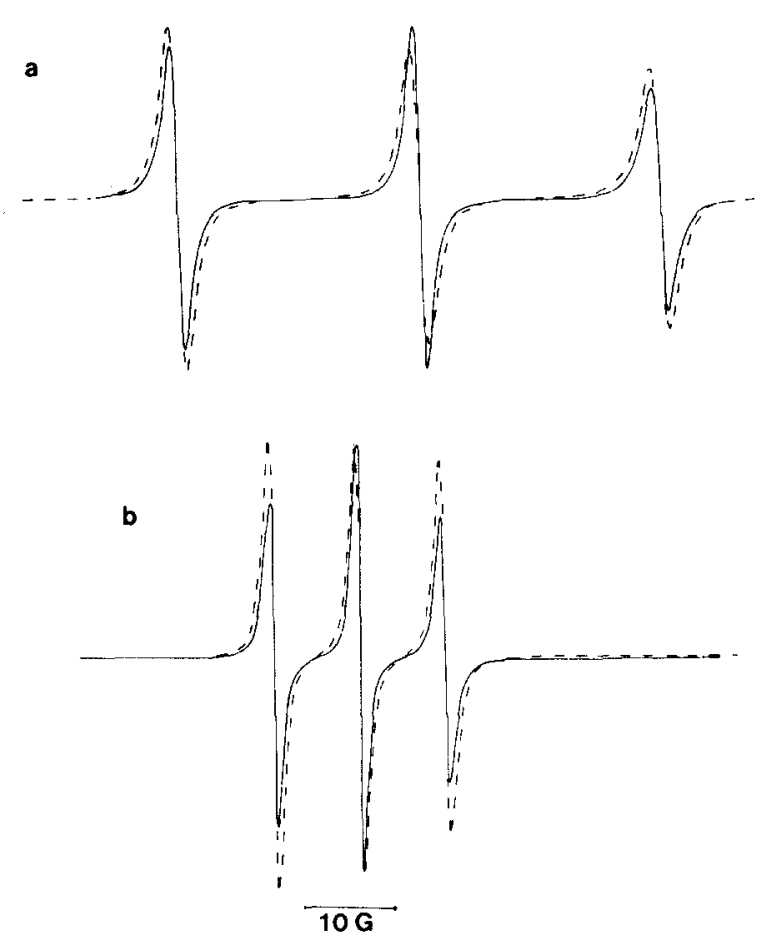

FIG. 5. Influence of the pseudosecular terms. Same parameters as in Fig. 1. Solid line: pseudosecular terms neglected. Broken line: pseudosecular terms included [corresponds to Fig. 1(b) and $2(\mathrm{~b})$ ]. Spectra calculated for (a) $H_{0}$ parallel to $N$; (b) $H_{0}$ perpendicular to $N$. 
$\mathrm{cm}^{2} \mathrm{sec}^{-1}$. These results are in agreement with membrane viscosities and lateral diffusion constants as determined by entirely different methods. ${ }^{26-29}$

So far we have concentrated upon the dynamics of the bilayer molecules. Let us now discuss some structural aspects of the double layer. It should be pointed out that the line shape analysis confirms two experimental results already obtained previously.

(1) The order parameters $S_{1}$ and $S_{2}$ are not equal for label I(13.2), ${ }^{2}$ and (2) the ordering of the lipid molecules in the bilayer is sensitive to changes in temperature. ${ }^{8}$ Molecular motions within the hydrocarbon chain increase gradually as the temperature increases, leading to smaller order parameters. However, the line shape analysis also sheds some new light on the flexibility problem. It is found in our investigation that within the range of existence of the liquid crystalline bilayer $\left(45\right.$ to $\left.8{ }^{\circ} \mathrm{C}\right)$ the temperature behavior of the order parameters $S_{3}$ and $S_{1}$ can be explained by constant energy parameters $q_{3}$ and $q_{1}$. This gives us confidence not only that the probability function (5) is a reasonable
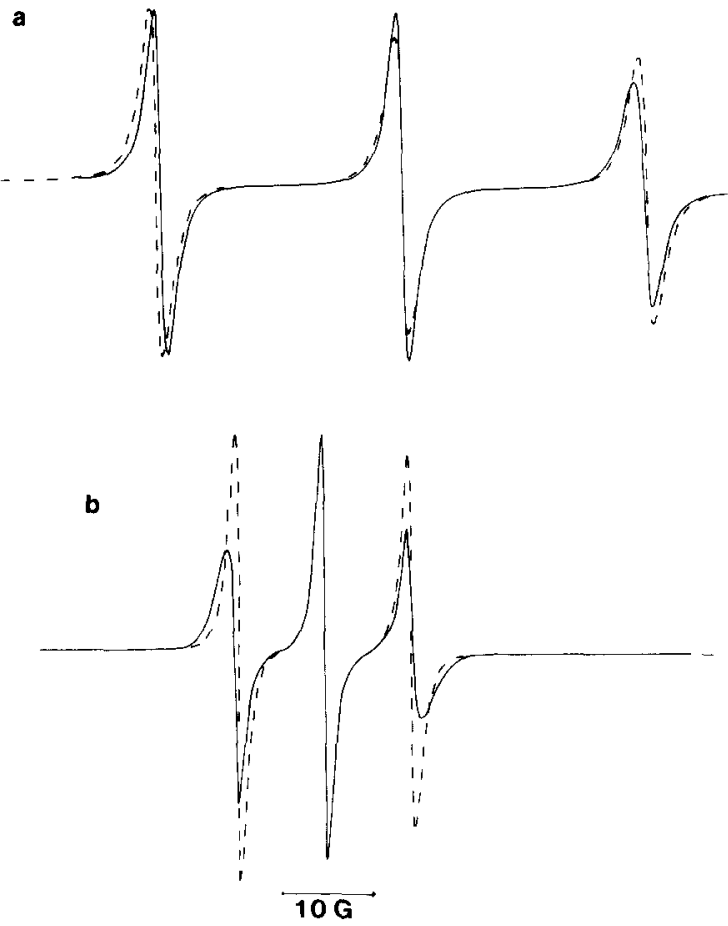

FIG. 6. Influence of the tilt angle. Same parameters as in Fig. 1. Solid line: $\bar{v}=10^{\circ}, \vartheta_{0}=5^{\circ}$. Broken line: $\vec{\vartheta}=0^{\circ}, \vartheta_{0}=3.4^{\circ}$ [corresponds to Figs. 1(b) and $2(\mathrm{~b})$ ]. Spectra calculated for (a) $H_{0}$ parallel to $N$; (b) $H_{0}$ perpendicular to $N$.
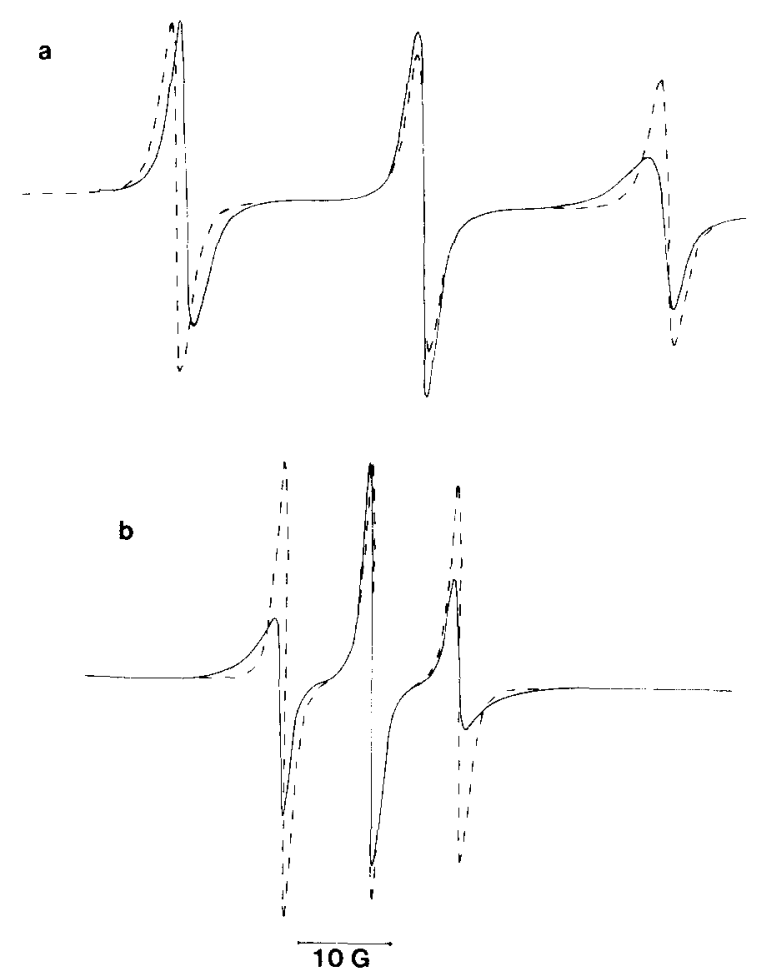

FIG. 7. Influence of the spread angle. Same parameters as in Fig. 1. Solid line: $\vartheta_{0}=15^{\circ}$. Broken line: $\vartheta_{0}=3.4^{\circ}$ [corresponds to Figs. 1(b) and 2(b)]. Spectra calculated for (a) $H_{0}$ parallel to $N$; (b) $H_{0}$ perpendicular to $N$.

choice for lipid bilayers but also that the very precise knowledge of the energy parameters $q$ may eventually open a way to a detailed statistical-mechanical understanding of bilayer structures. Even though this problem requires additional work, the description of the spin label spectra over a range of temperatures with only three parameters $\left(q_{1}\right.$, $q_{3}$, and $\left.\tau_{20}\right)$ is in itself a satisfying result.

The influence of tilt and spread angle on the spin label spectra of plamar oriented bilayers is illustrated in Fig. 6 and Fig. 7, respectively. Both parameters cause asymmetric broadening of the outer lines and reduce their amplitude relative to the central line. Furthermore, the positions of the outer lines in the spectrum are shifted. If the magnetic field $H_{0}$ is parallel (perpendicular) to the axis $N$ the calculated splittings in Fig. 6 and 7 are smaller (larger) compared to those calculated without tilt or spread angle. The latter spectra, which correspond to Figs. 1(b) and 2(b) are also included in Figs. 6 and 7. Comparing Figs. 1, 6, and 7 it is obvious that the occurrence of a tilt or spread angle in a lipid bilayer can be detected without difficulties. However, a very careful line shape analysis is required in order to distinguish between 


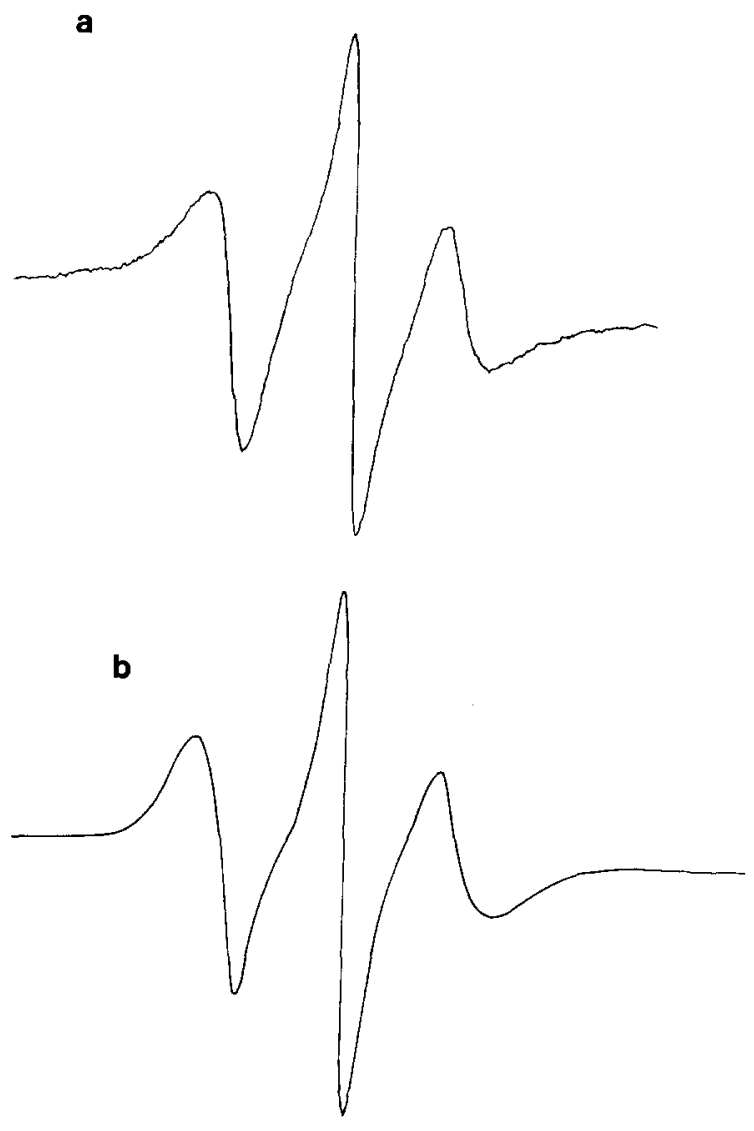

FIG. 8. Spin label $\mathrm{I}(13.2)$ in a planar oriented sample of bilayers at $-8^{\circ} \mathrm{C}$. Magnetic field $H_{0}$ perpendicular to bilayer normal $N$. (a) experimental spectrum, (b) calculated spectrum with $q_{3}=3.016 \mathrm{kcal} / \mathrm{mole}, q_{1}=-1.390$ $\mathrm{kcal} / \mathrm{mole} \tau_{20}=2.8 \cdot 10^{-9} \mathrm{sec}, \bar{\vartheta}=18^{\circ}, \vartheta_{0}=6^{\circ}$. Residual linewidth: $b=0.7 \mathrm{G}, c=0.8 \mathrm{G}$.

the two parameters, since the spectral changes caused by a tilt or spread angle are quite similar.

In the sodium decanoate-decanol bilayer, zero tilt angle and only a small spread angle are found in the range of 44 to $8{ }^{\circ} \mathrm{C}$. The axis of motional averaging is therefore perpendicular to the bilayer surface. This situation changes if the temperature is lowered below $8^{\circ} \mathrm{C}$. The spin label spectra then indicate that the liquid crystalline bilayer is transformed into a gel like structure with stiff hydrocarbon chains. This phase transition expresses itself not only in the energy parameters $q_{i}$ and the significant increase of the correlation time $\tau_{20}$, but it is also found that the fatty acid chains become tilted with respect to the bilayer normal. The tilt angle increases gradually with decreasing temperature. At $-8{ }^{\circ} \mathrm{C}$ the line shape analysis yields a tilt angle of $18^{\circ} \mathrm{C}$. The corresponding spectra are shown in Fig. 8 . Since the correlation time is already at lower limit for the applica- bility of our theory we cannot decide if the tilt angle further increases at even lower temperatures. Besides the spin label data we have also obtained calorimetric evidence for the observed phase transition.

In concluding, it should be emphasized that the theory of the spin label spectra presented here makes no assumptions about the specific motion of the spin label. In particular the order parameters should not be confused with any random walk model. ${ }^{30}$ The order parameters are an unambiguous and most general description for the averaging of tensorial quantities and need not further molecular foundation.

The purpose of this report is to present a general theory for the simulation of spin label spectra in liquid crystalline bilayers. Only a few examples have been given to illustrate the applicability of the method. The results of a systematic study of phospholipids and other membrane models by means of a variety of different spin labeled fatty acids will be published elsewhere.

\section{ACKNOWLEDGMENTS}

The authors are much indebted to Dr. B. Kowert for valuable comments and to Dr. C. Scandella for critically reading the manuscript.

*To whom correspondence should be addressed.

${ }^{1}$ D. Branton and D. N. Deamer, Membrane Structure (Springer, New York, 1972).

${ }^{2}$ J. Seelig, J. Am. Chern. Soc. 92, 3881 (1970).

${ }^{3}$ W. L. Hubbell and H. M. McConnell, J. Am. Chem. Soc. 93, 314 (1971).

${ }^{4}$ S. Rottem, W. L. Hubbell, L. Hayflick and H. M. McConnell, Biochim. Biophys. Acta 219, 104 (1970).

${ }^{5}$ J. Seelig and W. Hasselbach, Eur. J. Biochem. 21, 17 (1971).

${ }^{6}$ B. G. McFarland and H. M. McConnell, Proc. Natl. Acad. Sci. USA 68, 1274 (1971).

${ }^{7}$ H. M. McConnell and B. G. McFarland, Ann. N.Y. Acad. Sci. 195, 207 (1972).

${ }^{8}$ J. Seelig, J. Am. Chem. Soc. 93, 5017 (1971).

${ }^{9}$ J. Seelig, H. Limacher, and P. Bader, J. Am. Chem. Soc. 94, 6364 (1972).

${ }^{10} \mathrm{H}$. Limacher and J. Seelig, Angew. Chem. 84, 950 (1972); Angew. Chem. (Int. Ed. Engl.) 11, 920 (1972).

${ }^{11} \mathrm{~J}$. Seelig, F. Axel, and H. Limacher, "Molecular Architecture of Bilayer Membranes," Ann. N.Y. Acad. Sci. (to be published).

${ }^{12}$ A. Saupe, Z. Naturforsch. 19a, 161 (1964).

${ }^{13}$ H. Lippmann, Ann. Phys. (Leipz.) 2, 287 (1958).

${ }^{14}$ G. Meier and A. Saupe, Molec. Crystals 1, 515 (1966).

${ }^{15} \mathrm{~S}$. H. Glarum and J. H. Marshall, J. Chem. Phys. 46, 55 (1967).

${ }^{16}$ P. Diehl and C. F. Schwerdtfeger, Mol. Phys. 17, 423 (1969)

${ }^{17}$ J. H. Freed and G. K. Fraenkel, J. Chem. Phys. 39, 326 (1963).

${ }^{18}$ J. H. Freed, J. Chem. Phys. 41, 2077 (1964). 
${ }^{19}$ S. H. Glarum and J. H. Marshail, J. Chem. Phys. 44, 2884 (1966).

${ }^{20}$ G. R. Luckhurst and A. Sanson, Mol. Phys. 24, 1297 (1972).

${ }^{21}$ M. E. Rose, Elementary Theory of Angular Momentum (Wiley, New York, 1957).

${ }^{22}$ S. A. Goldman, G. V. Bruno, C. F. Polnaszek, and J. H. Freed, J. Chem. Phys. 56, 726 (1972).

${ }^{23} \mathrm{C}$. L. Hamilton and H. M. McConnell, Structural Chemistry and Molecular Biology, edited by A. Rich and N. Davidson (Freeman, San Francisco, 1968).

${ }^{24}$ L. J. Libertini and O. H. Griffith, J. Chem. Phys. 53, 1359 (1970).
${ }^{25}$ A. L. Buchachenko, A. M. Wasserman, and A. L. Kovarskii, Int. J. Chem. Kinet. 1, 361 (1969).

${ }^{26}$ R. A. Cone, Nat. New Biol. 236, 39 (1972).

${ }^{27}$ P. Deveaux and H. M. McConnell, J. Am. Chem. Soc.

94, 4475 (1972).

${ }^{28}$ E. Sackmann and H. Träuble, J. Am. Chem. Soc. 94, 4482 (1972); J. Am. Chem. Soc. 94, 4492 (1972); J. Am. Chem. Soc. 94, 4499 (1972).

${ }^{29}$ C. J. Scandella, P. Deveaux, and H. M. McConnell, Proc. Natl. Acad. Sci. USA 69, 2056 (1972).

${ }^{30}$ P. Jost, L. J. Libertini, V. C. Hebert, and O. H. Griffith, J Mol. Biol. 59, 77 (1971). 